\title{
A Study of the Validity of Performing Carotid Duplex Ultrasound with the Patient in a Seated Position
}

\author{
Lucia Pemble \\ School of Biomolecular and Physical Sciences, Griffith University, Nathan Campus, 170 Kessels Rd, Nathan, Brisbane, Qld 4111, \\ Australia
}

\begin{abstract}
Objective: The purpose of this study was to compare pulsed wave (PW) Doppler velocity measurements obtained from duplex ultrasound of the carotid arteries with subjects in the seated and supine positions in an attempt to determine if this would influence the velocity criteria used in the classification of the severity of internal carotid artery disease.

Methods: Carotid duplex ultrasound examinations were performed on 42 volunteers with measurement of peak systolic velocities (PSV) and end diastolic velocities (EDV) using a Doppler angle of $60^{\circ}$. The measurements were obtained from both the right and left extracranial carotid vessels in both the seated and supine positions. $t$-tests were used to compare the mean velocities obtained in the seated versus supine positions.

Results: PSV and EDV measurements obtained in the seated position were lower than those in the supine position. Statistical analysis comparing the means in the seated versus the supine position showed statistical significance at the $p=0.05$ level for all measurements except the PSV of the internal carotid artery (ICA).

Conclusions: Duplex ultrasound PW Doppler derived velocity criteria are used to classify the severity of stenosis, which in turn impacts on patient treatment options. The carotid duplex scan can be adequately performed on patients in the seated position but velocities measured will be reduced and modification to the existing velocity criteria may be needed.
\end{abstract}

Keywords: Internal Carotid Artery, Stenosis, Velocity Criteria, Duplex Ultrasound, Seated, Supine

\section{Introduction}

Carotid duplex ultrasound scanning is widely used in clinical practice for diagnosis of patients with symptoms of cerebrovascular disease. It has been extensively validated and is often the only diagnostic imaging performed before intervention. ${ }^{1,2}$ While all aspects of the carotid duplex ultrasound examination provide valuable information including vessel geometry, plaque characteristics and flow haemodynamics, it is often the peak systolic velocities (PSV), end diastolic velocities (EDV) and internal carotid artery (ICA) to common carotid artery (CCA) velocity ratios which categorise the severity of disease. ${ }^{3,4}$ There are numerous sets of published diagnostic criteria for the categorisation of severity of extracranial carotid disease and validation of criteria used within a facility is important. ${ }^{4,5}$ In addition, standardisation of protocol and adherence to criteria is crucial for accuracy and reproducibility of carotid duplex ultrasound results. ${ }^{4,6}$ Different pulsed wave (PW) Doppler insonation angles ${ }^{7}$ and gender differences ${ }^{8}$ have been reported to produce variation in PSV and EDV measurements. Clinical research supporting surgical benefit to patients with different categories of stenotic disease and symptomatic status emphasises the important role velocity criteria have in determining distinct categories of stenotic disease ${ }^{9}$ aiding clinical decision making for specific patients.

Carotid duplex ultrasound examination of the cerebrovasculature and angiographic studies used for correlation has been performed with subjects in the supine position.

Clinically, patients can present for carotid duplex ultrasound evaluation who may be unable to assume a supine position. They may be quadriplegic, suffer severe vertigo, spinal abnormalities or shortness of breath, and are restricted in their ability to assume a supine position for the scanning

Correspondence: L. Pemble, Senior Lecturer, School of Biomolecular and Physical Sciences, Griffith University, Nathan Campus, 170 Kessels Rd, Nathan, Brisbane, Qld 4111, Australia. I.pemble@griffith.edu.au procedure. This research investigates whether different carotid artery PSV, EDV and ICA/CCA PSV ratios are obtained when the carotid duplex ultrasound examination is performed with the patient in a seated position compared to those obtained in the supine position. I will then postulate if this would change the category of stenosis interpreted using the current velocity criteria for the classification of the severity of disease, as recommended by the Australasian Society of Ultrasound in Medicine ${ }^{10}$ or the Society of Radiologists in Ultrasound consensus conference recommendations. ${ }^{4}$

\section{Materials and Methods}

This research project was approved by the Griffith University Human Ethics Research Committee. All volunteers provided informed consent. There were 42 subjects ( 19 males and 23 females) with a mean age of 44 years (range: 19-70 years). All subjects were scanned by an accredited and experienced vascular sonographer (the author) in both the seated and supine positions. Subjects were randomly assigned to have their initial carotid duplex ultrasound scan performed in either the seated or supine position.

Colour duplex ultrasound examination of the right and left extracranial cerebrovascular system (common carotid, CCA; external carotid, ECA; internal carotid, ICA) was performed using the GE Logiq Book equipped with a 5-10 MHz linear array transducer (GE Healthcare, Australia). The initial scan plane used an anterolateral approach, which was optimised for each vessel and patient depending on vessel geometry, neck thickness and level of bifurcation. The B-mode image allowed visualisation of vessel characteristics and pathology while PW Doppler spectral traces were obtained using a Doppler angle of $60^{\circ}$ (to keep velocity errors consistent for all measurements). The PSV and EDV were obtained from the mid CCA, distal to the bulb of the ICA, or within the tightest region of stenosis (if present) 
Table 1. Mean and standard deviation (SD) values of $\mathrm{HR}$ and MAP from the 42 subjects measured from the right and left arms, in the seated and supine positions.

\begin{tabular}{lllllll}
\hline & \multicolumn{2}{l}{ Heart Rate $(\mathbf{b p m})$} & & \multicolumn{2}{l}{ Mean Arterial BP $(\mathbf{m m H g})$} \\
\cline { 3 - 3 } \cline { 5 - 6 } & & Mean & SD & & Mean & SD \\
\hline Right & Seated & 69.14 & 13.23 & & 93.11 & 10.30 \\
Right & Supine & 66.62 & 12.53 & & 90.17 & 11.57 \\
Left & Seated & 68.28 & 12.69 & & 91.41 & 10.51 \\
Left & Supine & 67.02 & 12.62 & & 89.78 & 11.05 \\
\hline
\end{tabular}

and within the proximal ECA. Sample volume was centrally placed, spectral Doppler scale and gain were adjusted to optimise the quality of the trace and measurements were obtained by visualisation and manual measurement from three consecutive cardiac cycles and then averaged.

Simultaneous blood pressure (BP) and heart rate (HR) measurements were obtained non-invasively (Omron IA2, Japan) ipsilateral to the carotid being investigated with duplex ultrasound. Three measurements of $\mathrm{BP}(\mathrm{mmHg})$ and $\mathrm{HR}(\mathrm{bpm})$ were obtained in each position on both the right and left side with the average being calculated. Mean arterial blood pressure (MAP) was calculated from the values of systolic and diastolic pressure measured using the equation MAP $(\mathrm{mmHg})=$ diastolic pressure $+1 / 3$ (systolic - diastolic pressure). ${ }^{11}$

Excel was used to store the data and perform descriptive statistics and $t$-tests comparing the mean velocities for the CCA, ICA and ECA in the different positions.

\section{Results}

The cardiovascular history of the 42 volunteers revealed six smokers, three ex-smokers, four hypertensive and three hypercholesterolaemic. None were diabetic or had a history of cerebrovascular accident, transient ischaemic attack or myocardial infarction. Owing to the small subject numbers exhibiting various cardiovascular risk factors, analysis of variance for individual factors was not performed and the data from all 42 volunteers were pooled for the following analysis. No subject was considered to have carotid arterial disease of greater than 50\% diameter reduction (DR) (using either the $\mathrm{ASUM}^{10}$ or the Society of Radiologists in Ultrasound ${ }^{4}$ criteria in the supine position).

The HR (bpm) and MAP ( $\mathrm{mmHg}$ ) measurements are shown for the right and left sides in both the seated and supine positions (Table 1). There were no statistical significant differences for HR or MAP between right and left sides or seated and supine positions.

The mean and standard deviation for PSV $(\mathrm{cm} / \mathrm{s})$ and EDV $(\mathrm{cm} / \mathrm{s})$ for each of the blood vessels (CCA, ICA and ECA) in both the seated and supine positions are shown in Table 2. All velocities measured in the seated position were less than those obtained in the supine position. Statistical analysis using the $t$-test, comparing the means in the seated versus supine position showed statistical significance at the $p=0.05$ level for all measurements except the PSV of the ICA.

The percentage change in velocities was calculated to compare the mean PSV and mean EDV in the seated position to that obtained in the supine position. The results are listed in Table 3 and show all velocities measured in the seated position were less than those obtained in the supine position, hence a negative percentage change.

If the percentage changes in PSV and EDV for the arteries in the seated position are applied to the ASUM velocity criteria and ratios listed in Table 4, the diagnostic criteria would change to ICA 50-69\% DR =PSV $>120 \mathrm{~cm} / \mathrm{s}$, ICA EDV $<$ $101 \mathrm{~cm} / \mathrm{s}$ and ICA/CCA PSV ratio $>2 \cdot 2$. Similarly applying the negative percentage change to velocities for the category of $70-79 \%$ DR will change the ICA PSV to $>259 \mathrm{~cm} / \mathrm{s}$, ICA EDV to $>101 \mathrm{~cm} / \mathrm{s}$ and the ICA/CCA PSV ratio to $>4.4$. For the 80-99\% DR category, the ICA PSV $>259 \mathrm{~cm} / \mathrm{s}$, ICA EDV $>$ $129 \mathrm{~cm} / \mathrm{s}$ and the ICA/CCA PSV ratio $>4.4$.

If the percentage changes in PSV and EDV are used to recalculate the Society of Radiologists in Ultrasound consensus data (listed in Table 4 in italics) the ICA/CCA PSV ratios are unchanged to those mentioned in the previous paragraph. Similarly classification of the $<50$ and $50-69 \%$ diameter reduction would be as above as the diagnostic criteria are identical. The difference between the two sets of criteria is in the $>70 \%$ diameter reduction category. The velocities suggestive of this degree of stenotic change would be ICA PSV of $>221 \mathrm{~cm} / \mathrm{s}$ and ICA EDV of $>91 \mathrm{~cm} / \mathrm{s}$. For tighter degrees of stenosis, there are no specific numerical values, which are quoted as being 'variable'. ${ }^{4}$

\section{Discussion}

Both carotid duplex ultrasound scanning and angiography are performed with the patient in a supine position. This raises the question 'is it valid to perform the carotid duplex examination in a seated position?'. Cardiovascular physiology involves integration of numerous components with regulation by the cardiovascular centre in the medulla oblongata maintaining homeostasis and control of mean arterial blood pressure. ${ }^{11}$ This is achieved via neural and hormonal regulation of the heart rate, stroke volume, peripheral resistance, mean arterial blood pressure and blood volume. In changing from a supine to a seated position, these cardioregulatory systems mediate the orthostatic response ensuring cardiac output and mean arterial pressure are maintained. In the cerebrovascular system, the aim is therefore to maintain cerebral blood flow. This research aims to provide information to determine if the current diagnostic velocity criteria can be accurately applied when the patient is in the seated position.

Performance of a carotid duplex ultrasound is technically possible on subjects in the seated position. It requires some additional hand-eye coordination and dexterity on behalf of the sonographer, but good quality images and PW Doppler spectral traces can be obtained from all the extracranial vessels

Table 2. Mean and standard deviation (SD) and $t$-test results comparing the mean PSV and EDV in the seated verses supine position for the CCA, ICA and ECA. Velocities were measured in units of $\mathrm{cm} / \mathrm{s}$.

\begin{tabular}{|c|c|c|c|c|c|c|}
\hline & CCA & CCA & ICA & ICA & ECA & ECA \\
\hline & $\mathrm{PSV}^{*}$ & $\mathrm{EDV}^{*}$ & PSV (nsd) & $\mathrm{EDV}^{*}$ & $\mathrm{PSV}^{*}$ & $\mathrm{EDV}^{*}$ \\
\hline Seated & $80 \cdot 08 \pm 17 \cdot 19$ & $19.75 \pm 4.06$ & $63.77 \pm 15.26$ & $22.61 \pm 4.89$ & $63.06 \pm 15.98$ & $7.57 \pm 6.68$ \\
\hline Supine & $90 \cdot 70 \pm 22.84$ & $22.55 \pm 4.58$ & $66.28 \pm 14.65$ & $24.62 \pm 5.52$ & $69.60 \pm 17.03$ & $14.34^{-} \pm 5.40$ \\
\hline
\end{tabular}

*Statistical significance at $p=0.05$

nsd: no significant difference. 
Table 3. Percentage change in velocity comparing the seated to the supine position for the PSV and EDV in the CCA, ICA and ECA. A negative percentage indicates that velocities in the seated position were less than those obtained in the supine position.

\begin{tabular}{ll}
\hline CCA PSV & $-11 \cdot 71 \%$ \\
CCA EDV & $-12 \cdot 42 \%$ \\
ICA PSV & $-3 \cdot 78 \%$ \\
ICA EDV & $-8 \cdot 19 \%$ \\
ECA PSV & $-9 \cdot 40 \%$ \\
ECA EDV & $-47 \cdot 17 \%$ \\
\hline
\end{tabular}

investigated. The MAP and HR measurements showed no change with position, supporting the well integrated orthostatic responses of the subjects. However, the results indicate differences in the carotid artery blood velocities obtained when the subject is seated, with all the velocities being reduced in this position compared with the velocities measured in the supine position. This velocity difference was not due to Doppler angle as this was constant at $60^{\circ}$. The PSV from the ICA was not statistically significantly different between the two positions, while all other PSV and EDV were significantly reduced in the seated position. The differences noted could be a reflection of the different resistances offered by the vascular beds fed by the extracranial carotid vessels. The higher resistance vascular bed of the face and scalp being supplied by the ECA shows a more significant decrease in PSV and EDV in the seated position. The constant velocity in the ICA suggests that the brain has finely tuned autoregulation of blood supply, which is well regulated to accommodate body position and has not been altered by body posture at the time of measurement

Our results suggest that the ICA PSV is still valid for a carotid duplex ultrasound examination performed on a patient in the seated position due to the sensitive homeostatic response of blood flow autoregulation within the brain. However, the EDV measured will be $\sim 10 \%$ less in this position. As ICA EDV is used to help determine the category of diameter reduction due to stenosis, then correction factors may be required. Similarly, the $10 \%$ reduction in CCA PSV measured with subjects in the seated position will have an impact on the ICA/CCA PSV ratio determined, especially in light of the fact that the ICA PSV is not significantly changed. This will result in higher ratios in the seated position ( $>2.2$ for $50-69 \%$ DR and $>4.4$ for $70-99 \%$ DR) equating with diameter reducing stenosis due to altered haemodynamics in this position. These changes are the same

Table 4. Carotid duplex ultrasound velocity criteria for the determination of the severity of stenosis (ASUM 2007). ${ }^{10}$

\begin{tabular}{llll}
\hline & \multicolumn{2}{l}{ PW Doppler criteria } & \\
\cline { 2 - 4 } Stenosis grade & PSV (cm/s) & EDV (cm/s) & ICA/CCA PSV ratio \\
\hline 0 & $<125$ & - & \\
& $<125$ & $<40$ & \\
$<15 \%$ DR & $<125$ & - & \\
$16-49 \%$ DR & - & - & \\
$50-69 \%$ DR & $<125$ & $<40$ & $>2$ \\
$70-79 \%$ DR & $>125$ & $<40$ & $>4$ \\
& $>125$ & $<110$ & $>4$ \\
$80-99 \%$ DR & $>270$ & $40-100$ & \\
& $>230$ & $>110$ & NA \\
Occluded & $>270$ & $>140$ & \\
& Variable & Variable & No flow \\
& No flow & No flow & \\
& & & \\
\hline
\end{tabular}

Society of Radiologists in Ultrasound criteria are expressed in italics. ${ }^{4}$ $\mathrm{DR}=$ diameter reduction. for the two different sets of diagnositic criteria studied. ${ }^{4,10}$ In this study, the subject sample consisted of only 42 subjects and none displayed haemodynaimcally significant ICA disease of greater than $50 \%$ diameter reduction. The application of these results to diameter reducing lesions in the higher categories is uncertain, but speculation could be made as to the need for some degree of adjustment to the velocity derived criteria and ratios.

While the $t$-tests showed statistically significant differences in some of the velocities measured in the seated position compared with the supine position, statistical significance does not necessarily indicate clinical significance. For some of the results, the difference between the groups is as large as the standard deviation surrounding the mean values. The validity of assumptions regarding the clinical significance of these differences requires further examination. It is necessary to both increase the subject number and ensure adequate representation of subjects in the tighter categories of disease.

\section{Conclusion}

This research suggests that it is feasible to perform the carotid duplex scan on patients in a seated position. While PSV values measured from the ICA remain valid, ICA EDV and ICA/CCA PSV ratios may need to be adjusted to prevent the misrepresentation of the degree of stenosis within the ICA. This could have important clinical implications for treatment regimes offered to these patients. Further research with more patients including representation from all categories of stenosis would be required to produce reader operator characteristic curves allowing establishment of new criteria.

\section{References}

1. Schneider PA, Silva MB, Jr, Bohannon WT, et al. Safety and efficacy of carotid arteriography in vascular surgery practice. J Vasc Surg 2005;41:238-245.

2. Langan EM, 3rd, Gray BH, Sullivan TM. Carotid angiography in contemporary vascular surgery practice. Semin Vasc Surg 2005; 18:83-86

3. Gaitine D, Soudack M. Diagnosing carotid stenosis by Doppler sonography. J Ultrasound Med 2005;24:1127-1136.

4. Grant EG, Benson CB, Moneta GL, et al. Carotid artery stenosis: grayscale and Doppler ultrasound diagnosis - Society of Radiologists in Ultrasound Consensus Conference. Ultrasound Q 2003;19:190-198.

5. Jahromi AS, Cina CS, Liu Y, Clase CM. Sensitivity and specificity of colour duplex ultrasound measurement in the estimation of internal carotid artery stenosis: a systematic review and metaanalysis. J Vasc Surg 2005;41:962-972.

6. Poe P, DeMasi RJ. Establishing interpretation criteria. J Vasc Ultrasound 2004;28:141-142.

7. Tola M, Yurdakul M. Effect of Doppler angle in diagnosis of internal carotid artery stenosis. J Ultrasound Med 2006;25:1187-1192.

8. Comerota AJ, Salles-Cunha SX, Daoud Y, Jones L, Beebe HG. Gender differences in blood velocities across carotid stenoses. J Vasc Surg 2004;40:939-944.

9. Neschis DG, Lexa FJ, Davis JT, Carpenter JP. Duplex criteria for determination of $50 \%$ or greater carotid stenosis. J Ultrasound Med 2001;20:207-215

10. Australian Society for Ultrasound in Medicine. Policies and Statements D14: colour duplex Doppler ultrasound extracranial carotid artery disease. Available at: http//www.asum.com.au/ open/home.htm

11. Levy MN, Pappano AJ. Cardiovascular physiology, 9th edn. St Louis, MO: Mosby, 2007. 